Atomistic simulation and continuum modeling of graphene nanoribbons under uniaxial tension

This article has been downloaded from IOPscience. Please scroll down to see the full text article.

2011 Modelling Simul. Mater. Sci. Eng. 19054006

(http://iopscience.iop.org/0965-0393/19/5/054006)

View the table of contents for this issue, or go to the journal homepage for more

Download details:

IP Address: 218.23.88.210

The article was downloaded on 23/06/2011 at 19:22

Please note that terms and conditions apply. 


\title{
Atomistic simulation and continuum modeling of graphene nanoribbons under uniaxial tension
}

\author{
Qiang Lu, Wei Gao and Rui Huang \\ Department of Aerospace Engineering and Engineering Mechanics, University of Texas at \\ Austin, Austin, TX 78712, USA
}

Received 5 January 2011, in final form 19 April 2011

Published 23 June 2011

Online at stacks.iop.org/MSMSE/19/054006

\begin{abstract}
Atomistic simulations are performed to study the nonlinear mechanical behavior of graphene nanoribbons under quasistatic uniaxial tension, emphasizing the effects of edge structures (armchair and zigzag, without and with hydrogen passivation) on elastic modulus and fracture strength. The numerical results are analyzed within a theoretical model of thermodynamics, which enables determination of the bulk strain energy density, the edge energy density and the hydrogen adsorption energy density as nonlinear functions of the applied strain based on static molecular mechanics simulations. These functions can be used to describe mechanical behavior of graphene nanoribbons from the initial linear elasticity to fracture. It is found that the initial Young's modulus of a graphene nanoribbon depends on the ribbon width and the edge chirality. Furthermore, it is found that the nominal strain to fracture is considerably lower for graphene nanoribbons with armchair edges than for ribbons with zigzag edges. Molecular dynamics simulations reveal two distinct fracture nucleation mechanisms: homogeneous nucleation for the zigzag-edged graphene nanoribbons and edgecontrolled heterogeneous nucleation for the armchair-edged ribbons. The modeling and simulations in this study highlight the atomistic mechanisms for the nonlinear mechanical behavior of graphene nanoribbons with the edge effects, which is potentially important for developing integrated graphene-based devices.
\end{abstract}

(Some figures in this article are in colour only in the electronic version)

\section{Introduction}

Graphene ribbons with nanoscale widths $(W<20 \mathrm{~nm}$ ) have been produced recently, either by lithographic patterning [1-3] or by chemically derived self-assembly processes [4], with potential applications in nanoelectronics and electromechanical systems. The edges of graphene nanoribbons (GNRs) can be zigzag, armchair or a mixture of both [5]. It has been theoretically predicted that the special characteristics of the edge states lead to a size effect in the electronic state of graphene and control whether the GNR is metallic, insulating 
or semiconducting [5-8]. The effects of edge structures on deformation and mechanical properties of GNRs have also been studied to some extent [9-18]. On the one hand, elastic deformation of GNRs has been suggested as a viable method to tune the electronic structure and transport characteristics in graphene-based devices $[15,16]$. On the other hand, plastic deformation and fracture of graphene may pose a fundamental limit for reliability of integrated graphene structures.

The mechanical properties of bulk graphene (i.e. infinite lattice without edges) have been studied both theoretically [19-21] and experimentally [22]. For GNRs, however, various edge structures are possible [23, 24], with intricate effects on the mechanical properties. Ideally, the mechanical properties of GNRs may be characterized experimentally by uniaxial tension tests. To date, however, no such experiment has been reported, although similar tests were performed for carbon nanotubes (CNTs) [25]. Theoretically, previous studies on the mechanical properties of GNRs have largely focused on the linear elastic properties (e.g. Young's modulus and Poisson's ratio) [11-15]. While a few studies have touched upon the nonlinear mechanical behavior including fracture of GNRs $[12,13,16]$, the effect of edge structures in the nonlinear regime has not been well understood. In this study, by combining atomistic simulations with a thermodynamics-based continuum model, we systematically investigate the nonlinear elastic deformation of GNRs under quasistatic uniaxial tension, emphasizing the effects of edge structures in both linear and nonlinear regimes.

The paper is organized as follows. Section 2 describes the method of atomistic simulations. A thermodynamics model is presented in section 3 for analysis of the numerical results. Section 4 discusses the edge effect on initial Young's modulus of GNRs, and section 5 discusses fracture of graphene. In section 6, the effect of hydrogen adsorption is analyzed. Section 7 summarizes the results.

\section{Atomistic simulation}

The second-generation reactive empirical bond-order (REBO) potential [26] is used in this study for atomistic simulations. Briefly, the potential energy of an atomistic system is calculated as

$$
\Phi=\sum_{i} \sum_{j>i}\left[V_{\mathrm{R}}\left(r_{i j}\right)-\bar{b}_{i j} V_{\mathrm{A}}\left(r_{i j}\right)\right],
$$

where $r_{i j}$ is the interatomic distance between atoms $i$ and $j, V_{\mathrm{R}}$ and $V_{\mathrm{A}}$ are pairwise potential functions for the repulsive and attractive interactions, respectively, and $\bar{b}_{i j}$ is a bond-order term that depends on the number and types of neighbors to account for many-body interactions. In particular, the bond-order function, $\bar{b}_{i j}$, in the second-generation REBO potential takes into account the local bonding environment up to the third nearest neighbors, through its dependence on both bond angles and dihedral angles [27]. With this, the REBO potential allows the influence of atomic re-hybridization on the binding energy to change as chemical bonds break and reform over the course of atomistic simulation. The complete form of the REBO potential for both carbon-carbon $(\mathrm{C}-\mathrm{C})$ and carbon-hydrogen $(\mathrm{C}-\mathrm{H})$ interactions is given in [26].

To limit the range of covalent interactions, a cutoff function is typically used in atomistic simulations. The originally suggested form of the cutoff function for the REBO potential is

$$
f_{\mathrm{c}}(r)=\left\{\begin{array}{ll}
1 & \begin{array}{l}
1 \\
\frac{1}{2}
\end{array}\left[1+\cos \left(\frac{\left(r-D_{1}\right) \pi}{D_{2}-D_{1}}\right)\right] \\
0 & D_{1}<r<D_{2} \\
& r>D_{2}
\end{array}\right\},
$$


(a)
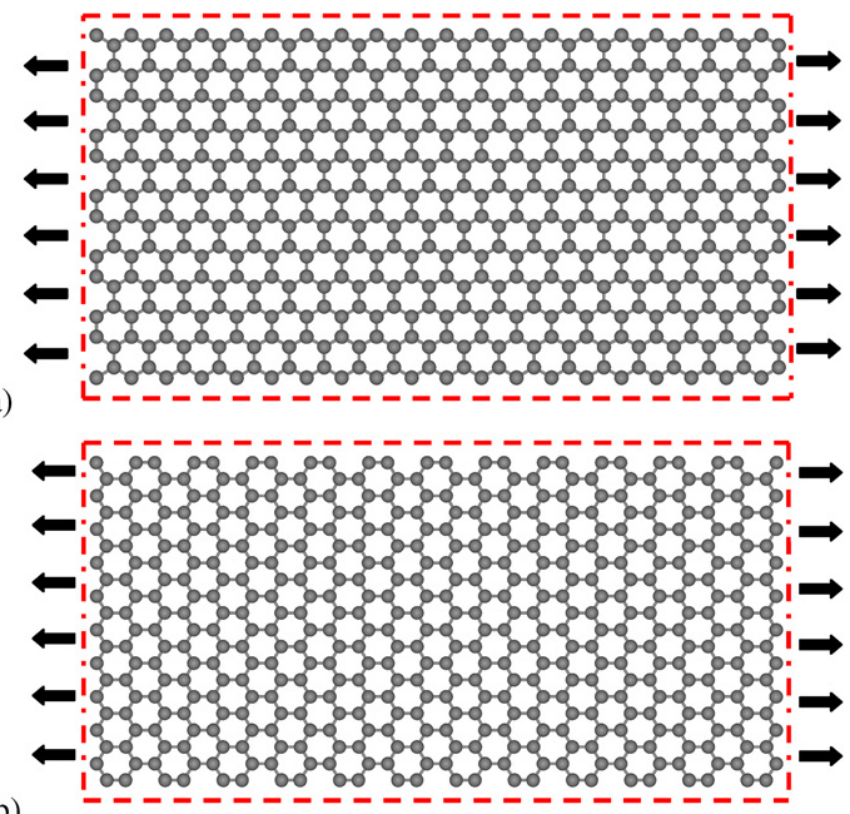

Figure 1. Rectangular GNRs with $(a)$ zigzag and $(b)$ armchair edges, subjected to uniaxial tension.

where $D_{1}$ and $D_{2}$ are the two cutoff distances for a smooth transition from 1 to 0 as the interatomic distance $(r)$ increases. For $\mathrm{C}-\mathrm{C}$ interaction, $D_{1}=1.7 \AA$ and $D_{2}=2.0 \AA$ were suggested [26]. However, as noted in several previous studies [12,28-30], such a cutoff function typically generates spurious bond forces near the cutoff distances, an unphysical result due to discontinuity in the second derivative of the cutoff function. This artifact shall be avoided in the study of nonlinear mechanical properties of graphene under relatively large strains. As suggested by the developers of the original REBO potential [28], using a larger cutoff distance could remove the unphysical responses. However, to keep the pair interactions within the nearest neighbors, the cutoff distance must not be too large. In this study, the cutoff function is taken to be 1 within a cutoff distance $\left(D_{1}=1.9 \AA\right)$ and zero otherwise. It is found that the numerical results up to fracture of GNRs are unaffected by the choice of the cutoff distance within the range between 1.9 and $2.2 \AA$.

Classical molecular mechanics (MM) simulations are performed for GNRs subjected to quasistatic uniaxial tension. For each MM simulation, a rectangular GNR of width $W$ and length $L$ is first cut out from the ground state of an infinite graphene lattice, as shown by two examples in figure 1. Next, by holding the length of the GNR with periodic boundary conditions at both ends, edge relaxation is simulated to obtain the equilibrium state of the GNR at zero strain $(\varepsilon=0)$. As shown in a previous study [18], the ribbon width reduces slightly upon edge relaxation. Subsequently, by gradually increasing the ribbon length, a longitudinal tensile strain $(\varepsilon>0)$ is applied. At each strain level, the statically equilibrium lattice structure of the GNR is calculated to minimize the total potential energy by a quasiNewton algorithm [31]. For each GNR, the average potential energy per carbon atom at the equilibrium state is calculated as a function of the nominal strain until it fractures, as shown in figure 2. In all simulations, periodic boundary conditions are applied at both ends of the GNR, whereas the two parallel edges (zigzag or armchair) of the GNR are free of external 

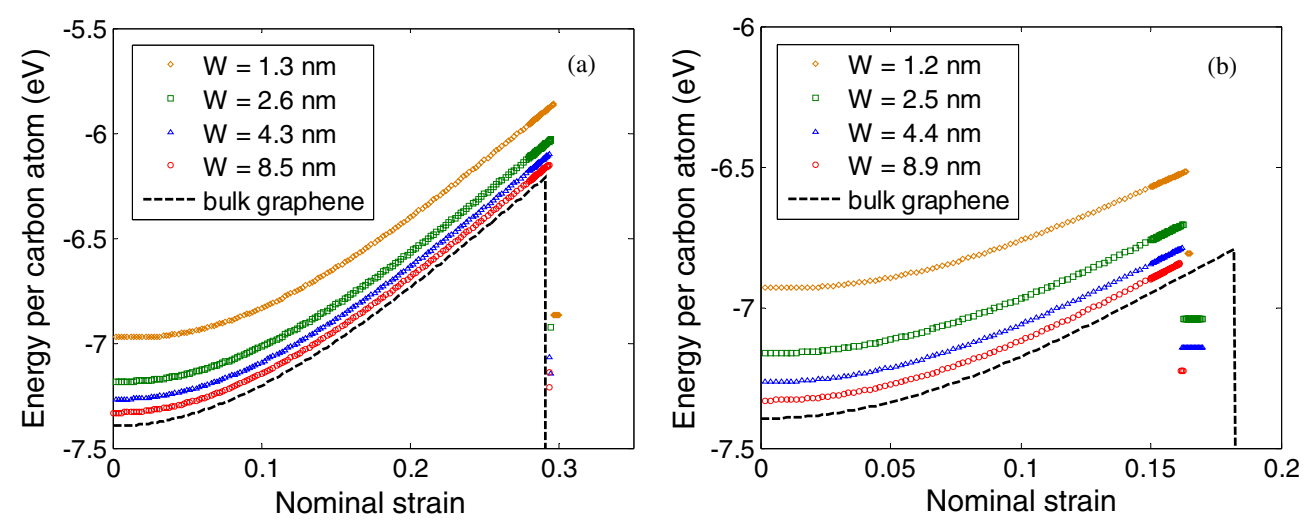

Figure 2. Potential energy per carbon atom as a function of the nominal strain for GNRs under uniaxial tension, with $(a)$ zigzag and $(b)$ armchair edges, both unpassivated. The dashed lines show the results for bulk graphene under uniaxial tension in the zigzag and armchair directions.

constraint. For comparison, the mechanical behavior of infinite graphene lattice under uniaxial tension is also simulated by applying periodic boundary conditions at all four edges, in which lateral relaxation perpendicular to the loading direction is allowed in order to achieve the uniaxial stress condition. To study the effect of hydrogen adsorption along the free edges, MM simulations of GNRs with both bare and hydrogen-passivated edges are performed.

The critical strain (or stress) to fracture as predicted by the static MM simulations may be considered the ideal strength of the defect-free GNRs at zero temperature ( $T=0 \mathrm{~K})$. However, the process of fracture nucleation and crack growth are typically not observable in the MM simulations. On the other hand, molecular dynamics (MD) simulations at finite temperatures can be used to study the fracture process. In this study, to qualitatively understand the fracture mechanisms, classical MD simulations of GNRs under uniaxial tension are performed at relatively low temperatures (from 0.1 to $300 \mathrm{~K}$ ). The temperature control is achieved using an Anderson thermostat [32]. Each GNR is loaded by increasing the nominal strain, with a dwelling period of about 2 ps (or 2000 time steps) at each strain level. The strain increment is adjusted so that increasingly smaller increments are used as the total strain increases, with a minimum increment at 0.0005 . The velocity-Verlet scheme is used for time integration with a time step of around $1 \mathrm{fs}$. We note that MD simulations are often sensitive to the temperature control and the loading rate. In this study, the MD simulations provide a qualitative understanding of the fracture mechanisms, consistent with the static MM calculations. The quantitative nature of the MD simulation is not essential for this purpose.

\section{Thermodynamics}

To understand the numerical results from atomistic simulations, we adopt a simple thermodynamics model for GNRs under uniaxial tension. For a GNR of width $W$ and length $L$, the total potential energy as a function of the nominal strain consists of contributions from deformation of the interior lattice (i.e. the bulk strain energy) and from the edges (i.e. the edge energy), namely

$$
\Phi(\varepsilon)=N U_{0}+U(\varepsilon) W L+2 \gamma(\varepsilon) L,
$$

where $\varepsilon$ is the nominal strain in the longitudinal direction of the ribbon (relative to the bulk graphene lattice at the ground state), $U_{0}$ is the potential energy per carbon atom at the ground 

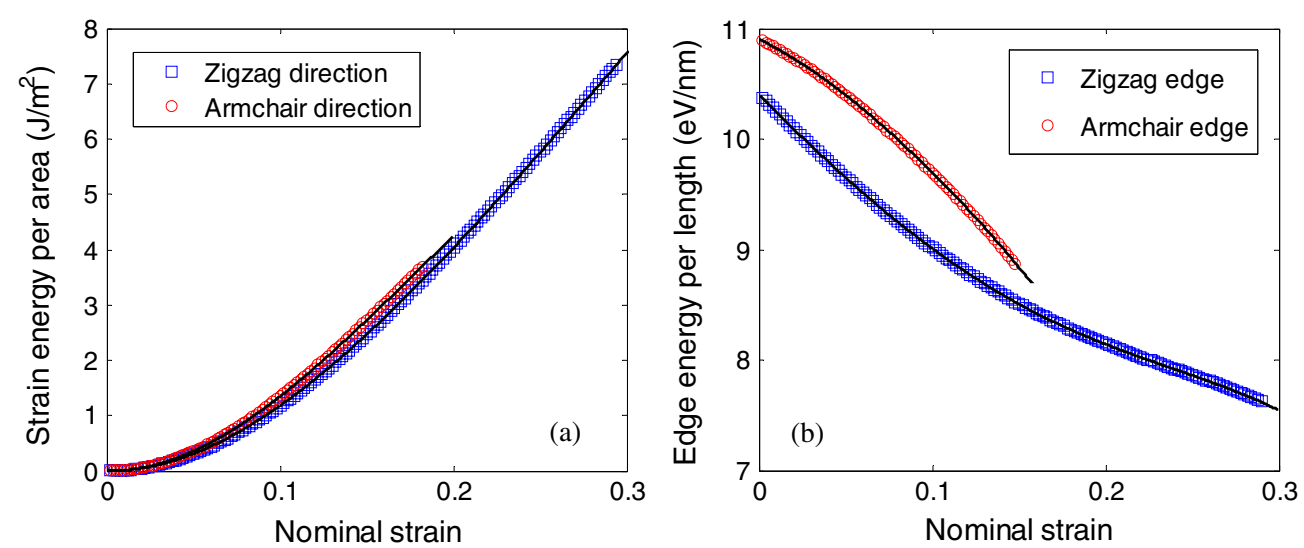

Figure 3. (a) Bulk strain energy density of monolayer graphene under uniaxial tension in the zigzag and armchair directions; $(b)$ edge energy density of GNRs under uniaxial tension. The open symbols are obtained directly from the atomistic simulations, and the solid lines are the polynomial functions in equations (6) and (8).

state of graphene, $N$ is the number of carbon atoms, $U(\varepsilon)$ is the bulk strain energy density of monolayer graphene (per unit area) and $\gamma(\varepsilon)$ is the edge energy density (per unit length of the free edges). The average potential energy per carbon atom is thus

$$
\bar{\Phi}(\varepsilon)=\frac{\Phi(\varepsilon)}{N}=U_{0}+U(\varepsilon) A_{0}+\frac{2 A_{0}}{W} \gamma(\varepsilon),
$$

where $A_{0}=\frac{3}{4} \sqrt{3} r_{0}^{2}$ is the area per carbon atom at the ground state of graphene and $r_{0}=1.42 \AA$ is the equilibrium bond length of graphene. As shown in figure 2, the average potential energy increases as the ribbon width $(W)$ decreases, an effect due to the contribution of the edge energy (i.e. the third term on the right-hand side of equation (4)).

For an infinite graphene monolayer $(W \rightarrow \infty)$, the bulk strain energy density function, $U(\varepsilon)$, can be obtained directly from the MM calculations, namely

$$
U(\varepsilon)=\frac{\bar{\Phi}(\varepsilon ; W \rightarrow \infty)-U_{0}}{A_{0}} .
$$

Figure 3(a) shows the calculated bulk strain energy density versus the nominal strain in the zigzag and armchair directions. For each case, the numerical results from atomistic simulations are fitted with a polynomial function up to eighth order of the nominal strain, namely

$$
U(\varepsilon)=a_{2} \varepsilon^{2}+a_{3} \varepsilon^{3}+a_{4} \varepsilon^{4}+a_{5} \varepsilon^{5}+a_{6} \varepsilon^{6}+a_{7} \varepsilon^{7}+a_{8} \varepsilon^{8},
$$

where the coefficients are listed in table 1. The eighth-order polynomial function in (6) is necessary to achieve a satisfactory fitting with the second derivative of the strain energy density function. The leading term of the polynomial function is necessarily quadratic so that the strain energy is zero and a minimum at the ground state $(\varepsilon=0)$. Furthermore, the hexagonal symmetry of the graphene lattice at the ground state dictates that it is isotropic under an infinitesimal strain $(\varepsilon \ll 1)$. Thus, the quadratic term in equation (6) is independent of the loading direction. However, the symmetry is broken under a finite deformation, leading to nonlinear, anisotropic elastic properties [19-21], as represented by the high-order terms on the right-hand side of equation (6). Consequently, the coefficients listed in table 1 are different for the two loading directions except for the quadratic term $\left(a_{2}\right)$. 
Table 1. Coefficients of the polynomial fitting in equation (6) for the bulk strain energy density function of graphene subject to uniaxial tension in zigzag and armchair directions (unit: $\mathrm{J} \mathrm{m}^{-2}$ ).

\begin{tabular}{lll}
\hline & Zigzag & Armchair \\
\hline$a_{2}$ & 121.65 & 121.65 \\
$a_{3}$ & 144.06 & 1175.81 \\
$a_{4}$ & -2947.21 & -23584.89 \\
$a_{5}$ & 14517.28 & 219264.35 \\
$a_{6}$ & -41544.88 & -1189116.03 \\
$a_{7}$ & 66883.97 & 3459762.95 \\
$a_{8}$ & -46193.34 & -4159339.72 \\
\hline
\end{tabular}

Table 2. Coefficients of the polynomial fitting in equation (8) for the edge energy density of GNRs with zigzag and armchair edges (unit: $\mathrm{eV} \mathrm{nm}^{-1}$ ).

\begin{tabular}{lll}
\hline & Zigzag & Armchair \\
\hline$b_{0}$ & 10.41 & 10.91 \\
$b_{1}$ & -16.22 & -8.53 \\
$b_{2}$ & 25.99 & 11.39 \\
$b_{3}$ & -123.40 & -2034.17 \\
$b_{4}$ & 1387.77 & 37377.27 \\
$b_{5}$ & -6306.31 & -374309.95 \\
$b_{6}$ & 16090.44 & 2144425.42 \\
$b_{7}$ & -29257.32 & -6538094.57 \\
$b_{8}$ & 26649.06 & 8061231.96 \\
\hline
\end{tabular}

For GNRs, the edge energy density function is determined by subtracting the bulk energy from the total potential energy of the GNR based on equation (4), i.e.

$$
\gamma(\varepsilon)=\frac{W}{2 A_{0}}\left[\bar{\Phi}(\varepsilon)-U(\varepsilon) A_{0}-U_{0}\right] .
$$

Figure 3(b) shows the calculated edge energy density versus the nominal strain for the zigzag and armchair edges. The results are essentially independent of the ribbon width in the range considered for this study $(1 \mathrm{~nm}<W<10 \mathrm{~nm})$. Similar to the bulk strain energy density, a polynomial function up to eighth order of the nominal strain is used to fit the edge energy density, namely

$$
\gamma(\varepsilon)=b_{0}+b_{1} \varepsilon+b_{2} \varepsilon^{2}+b_{3} \varepsilon^{3}+b_{4} \varepsilon^{4}+b_{5} \varepsilon^{5}+b_{6} \varepsilon^{6}+b_{7} \varepsilon^{7}+b_{8} \varepsilon^{8},
$$

where the coefficients for the zigzag and armchair edges are listed in table 2 . The first term on the right-hand side of equation (8) is independent of the nominal strain, which represents the excess edge energy at zero strain $(\varepsilon=0)$ as discussed in the previous study [18]. The second term varies linearly with the strain, which gives the residual edge force or edge stress at zero strain [18]. In general, however, the edge energy is a nonlinear function of the nominal strain.

Next we consider variation of the potential energy. Under uniaxial tension, the GNR is subjected to a net force $(F)$ in the longitudinal direction. At each strain increment, the mechanical work done by the longitudinal force equals the increase in the total potential energy, which can be written in a variational form, i.e.

$$
\delta \Phi=F L \delta \varepsilon .
$$

Therefore, the force $(F)$ can be obtained from the derivative of the potential energy function, with which a two-dimensional (2D) nominal stress can be defined without ambiguity as the 

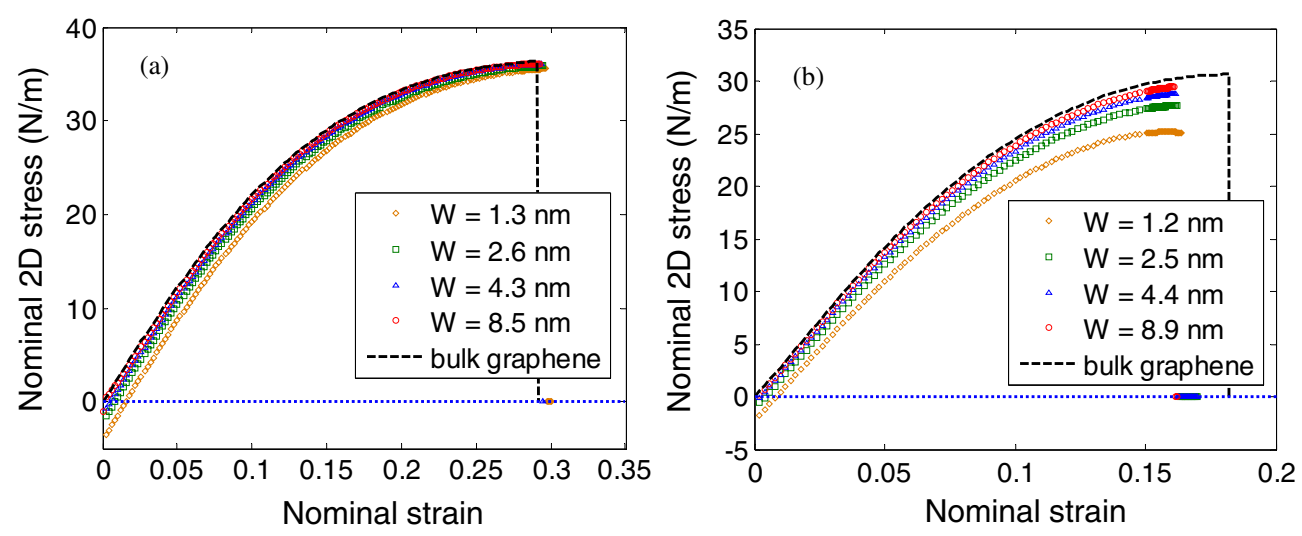

Figure 4. Nominal stress-strain curves for GNRs under uniaxial tension, with (a) zigzag and $(b)$ armchair edges, both unpassivated. The dashed lines show the results for bulk graphene under uniaxial tension in the zigzag and armchair directions.

force per unit width of the GNR, namely

$$
\sigma(\varepsilon)=\frac{F}{W}=\frac{\mathrm{d} U}{\mathrm{~d} \varepsilon}+\frac{2}{W} \frac{\mathrm{d} \gamma}{\mathrm{d} \varepsilon} .
$$

Note that we do not assume any specific thickness for the monolayer graphene in the definition of the 2D stress. When placed on a substrate, the thickness of a graphene monolayer depends on the interaction between graphene and the substrate [33], which is not an intrinsic property of graphene itself. As a result, the 2D stress in equation (10) has a unit of $\mathrm{N} \mathrm{m}^{-1}$, different from the conventional 3D stress $\left(\mathrm{N} \mathrm{m}^{-2}\right)$. Figure 4 shows the nominal stress-strain curves of the GNRs, obtained by numerically taking the derivative of the potential energy in figure 2 . Nearly identical stress-strain curves can be obtained analytically by equation (10) with the polynomial functions in equations (6) and (8). Apparently, the stress-strain relation for graphene is nonlinear in all cases, for which the tangent elastic modulus can be defined as

$$
E(\varepsilon)=\frac{\mathrm{d} \sigma}{\mathrm{d} \varepsilon}=\frac{\mathrm{d}^{2} U}{\mathrm{~d} \varepsilon^{2}}+\frac{2}{W} \frac{\mathrm{d}^{2} \gamma}{\mathrm{d} \varepsilon^{2}}
$$

For an infinite monolayer graphene $(W \rightarrow \infty)$, the stress-strain relation is fully determined by the bulk strain energy density function. With the polynomial function in equation (6), an analytical expression for the stress-strain relation may be obtained. In figure 5(a) we plot the stress-strain curves for infinite graphene subjected to uniaxial tension in the zigzag and armchair directions, comparing the results from the atomistic simulations with first-principles calculations by Wei et al [20]. Figure 5(b) shows the corresponding tangent modulus for bulk graphene. Apparently, the atomistic simulations with the REBO potential considerably underestimate the stiffness of the graphene monolayer, even under infinitesimal strain $(\varepsilon \sim 0)$. The initial Young's modulus, $E_{0}=(\mathrm{d} \sigma / \mathrm{d} \varepsilon)_{\varepsilon=0}$, is $243 \mathrm{~N} \mathrm{~m}^{-1}$ by the REBO potential and $345 \mathrm{~N} \mathrm{~m}^{-1}$ by the first-principles calculation. This discrepancy is the major shortcoming of the REBO potential in modeling mechanical behavior of graphene and CNTs, as noticed previously [34-36]. Nevertheless, the REBO potential has been used extensively, including this study, to qualitatively understand the mechanical behavior of low-dimensional carbon materials on the atomistic scale. Several modifications to the REBO potential have been suggested recently [37-39], which are yet to show consistent improvement in the prediction of Young's modulus of graphene. 

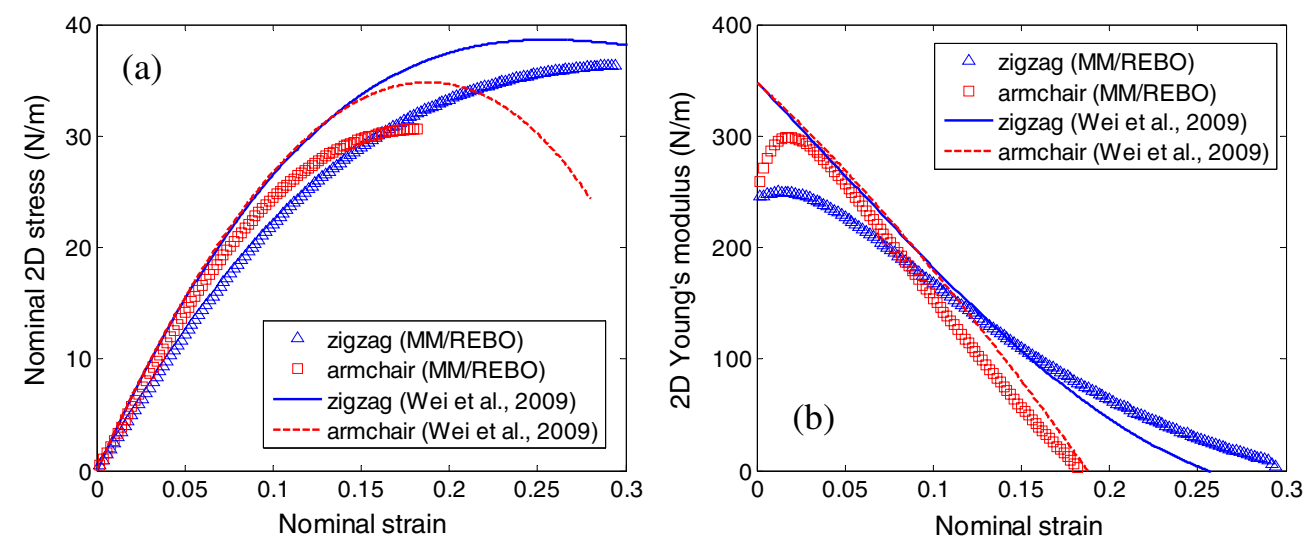

Figure 5. (a) Nominal stress-strain curves for monolayer graphene under uniaxial tension in the zigzag and armchair directions; $(b)$ tangent Young's modulus as a function of the nominal strain.

For GNRs, due to the edge effect, the nominal stress-strain relation depends on the ribbon width, as shown in figure 4. The difference between GNRs with zigzag edges and those with armchair edges is also appreciable, even at relatively small strains. We discuss the edge effects in the following sections.

\section{Edge effect on the initial Young's modulus}

The nominal stress-strain curves in figure 4 show approximately linear elastic behavior of all GNRs at relatively small strains (e.g. $\varepsilon<5 \%$ ). Following equation (11), the initial Young's modulus of the GNRs in the linear regime can be written as

$$
E_{0}=E_{0}^{\mathrm{b}}+\frac{2}{W} E_{0}^{\mathrm{e}}
$$

where $E_{0}^{\mathrm{b}}$ is the initial Young's modulus of bulk graphene and $E_{0}^{\mathrm{e}}$ is the initial edge modulus. Using the polynomial functions in equations (6) and (8), we have

$$
\begin{aligned}
& E_{0}^{\mathrm{b}}=\left(\frac{\mathrm{d}^{2} U}{\mathrm{~d} \varepsilon^{2}}\right)_{\varepsilon=0}=2 a_{2}, \\
& E_{0}^{\mathrm{e}}=\left(\frac{\mathrm{d}^{2} \gamma}{\mathrm{d} \varepsilon^{2}}\right)_{\varepsilon=0}=2 b_{2} .
\end{aligned}
$$

While bulk graphene is isotropic in the regime of linear elasticity, the initial edge modulus depends on the edge chirality with different values for the zigzag and armchair edges. As a result, the initial Young's modulus of the GNR depends on both edge chirality and ribbon width $(W)$, as shown in figure 6. The initial edge modulus obtained from the REBO potential in this study is $E_{0}^{\mathrm{e}}=8.33 \mathrm{nN}\left(\sim 52 \mathrm{eV} \mathrm{nm}^{-1}\right)$ for the unpassivated zigzag edge and $E_{0}^{\mathrm{e}}=3.65 \mathrm{nN}$ $\left(\sim 23 \mathrm{eV} \mathrm{nm}^{-1}\right)$ for the unpassivated armchair edge. With positive moduli for both edges, the Young's modulus of unpassivated GNRs increases as the ribbon width decreases. Figure 6 shows that the numerical results from the atomistic simulations agree closely with equation (12) using the polynomial fitting parameters for the bulk and edge modulus. As such, it is predicted that the edge effect on the initial Young's modulus of GNRs diminishes as the ribbon width increases. A similar effect has been reported for nanowires and nanofilms, for which the surface effect leads to size-dependent Young's modulus [40-42]. 


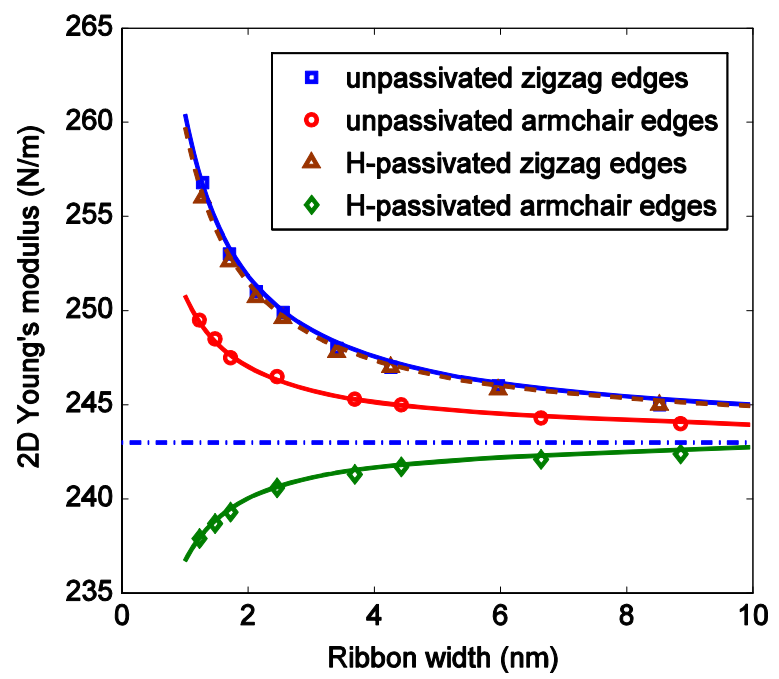

Figure 6. Initial Young's modulus versus ribbon width for GNRs with unpassivated and hydrogenpassivated edges. The horizontal dotted-dashed line indicates the initial Young's modulus of bulk graphene predicted by the REBO potential.

It is noted in figure 4 that the nominal stress is not zero for GNRs at zero nominal strain. This is due to the presence of a residual edge force (or edge stress) at zero strain. As discussed in the previous study [18], relaxation of the edge bonds results in a compressive edge force due to a mismatch in the equilibrium bond lengths. The edge force can be obtained as the first derivative of the edge energy function, namely

$$
f(\varepsilon)=\frac{\mathrm{d} \gamma}{\mathrm{d} \varepsilon} .
$$

With equation (8) for the edge energy density, the edge force at $\varepsilon=0$ equals the coefficient $b_{1}$, which is negative (compressive) for both zigzag and armchair edges as listed in table 2 . As a result, the nominal stress of the GNRs as defined in equation (10) is negative at zero strain and is inversely proportional to the ribbon width. The compressive edge force may lead to edge buckling [18], which would partly relax the nominal stress and potentially affect the initial stress-strain behavior for the GNRs. This effect is found to be negligible as the edge buckling is typically flattened under uniaxial tension with the nominal strain beyond a fraction of $1 \%$.

\section{Fracture of GNRs}

Without any defect, the theoretical strength of monolayer graphene (infinite lattice) is dictated by intrinsic lattice instability. As shown in several previous studies [19-21, 30,43], the critical strain to fracture for graphene varies with the loading direction. Under uniaxial tension, as shown in figure 5, the graphene monolayer fractures at the maximum nominal stress, when the tangent modulus becomes zero (i.e. $\mathrm{d}^{2} U / \mathrm{d} \varepsilon^{2}=0$ ). At a finite temperature, however, fracture may occur much earlier due to thermally activated processes [12]. It is noted that both the MM simulations and first-principles calculations predict higher tensile strength in the zigzag direction than in the armchair direction. However, the REBO potential underestimates the theoretical strength (fracture stress) of graphene in both directions. This discrepancy may be a result of the discrepancy in the predictions of the initial Young's modulus of graphene by the 

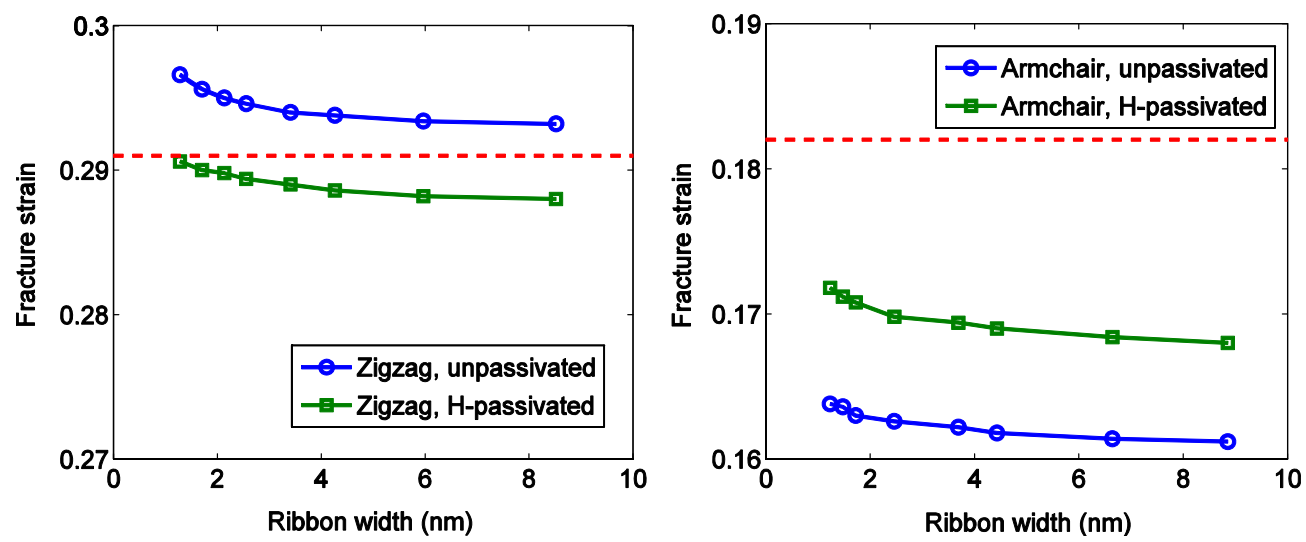

Figure 7. Fracture strain versus ribbon width for GNRs under uniaxial tension, with (a) zigzag and $(b)$ armchair edges. The horizontal dashed line in each figure indicates the fracture strain of bulk graphene under uniaxial tension in the same direction.

two methods. On the other hand, the REBO potential overestimates the fracture strain in the zigzag direction, whereas the predicted fracture strain in the armchair direction agrees closely with the first-principles calculation.

For GNRs, the lattice structure becomes inhomogeneous due to edge relaxation, which leads to two distinct fracture mechanisms for GNRs with zigzag and armchair edges. As shown in figure 4(a), the GNRs with zigzag edges fracture at a critical strain very close to that of bulk graphene loaded in the same direction. In contrast, figure 4(b) shows that the GNRs with armchair edges fracture at a critical strain considerably lower than bulk graphene. In both cases, the fracture strain slightly depends on the ribbon width, as shown in figure 7 . The apparently different edge effects on the fracture strain imply different fracture nucleation mechanisms for the zigzag- and armchair-edged GNRs, which are revealed by MD simulations.

To qualitatively understand the fracture processes of GNRs under uniaxial tension, MD simulations are performed at different temperatures $(0<T<300 \mathrm{~K})$. Figure 8 shows two examples of fractured GNRs at $50 \mathrm{~K}$. For the GNR with zigzag edges (figure $8(a)$ ), fracture nucleation occurs stochastically at the interior lattice of the GNR. As a result, the fracture strain is very close to that of bulk graphene strained in the same direction, consistent with the MM calculations (figure 7(a)). However, for the GNR with armchair edges (figure $8(b)$ ), fracture nucleation occurs exclusively near the edges. Thus, the armchair edge serves as the preferred location for fracture nucleation, leading to a considerably lower fracture strain compared with bulk graphene, as seen also from the MM calculations (figure $7(b)$ ). Therefore, two distinct fracture nucleation mechanisms are identified as interior homogeneous nucleation for the zigzag-edged GNRs and edge-controlled heterogeneous nucleation for the armchair-edged GNRs. In both cases, the fracture process is essentially brittle. The formation of suspended atomic chains is observable, mostly near the edges, in the MD simulations as shown in figure 8 . A similar chain formation was observed in experiments [44] and in a first-principles study [16].

It is evident from figure 8 that the cracks preferably grow along the zigzag directions of the graphene lattice in both cases. By the Griffith criterion for brittle fracture [45], this suggests lower edge energy in the zigzag direction of graphene as opposed to the armchair direction, which is consistent with our calculations of the edge energy in the previous study [18]. However, several first-principles calculations [17, 23, 46, 47] have predicted lower edge energy for the armchair edge, opposite to the calculations using empirical potentials [9, 18]. On the other hand, other first-principles calculations $[19,20]$ have predicted lower fracture strain and 
(a)

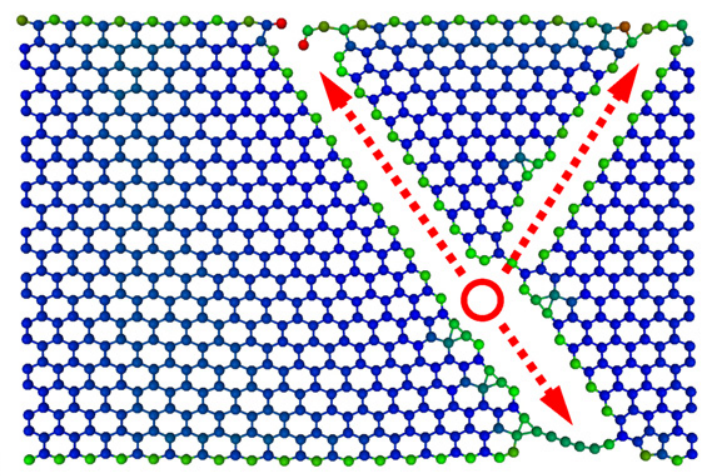

(b)

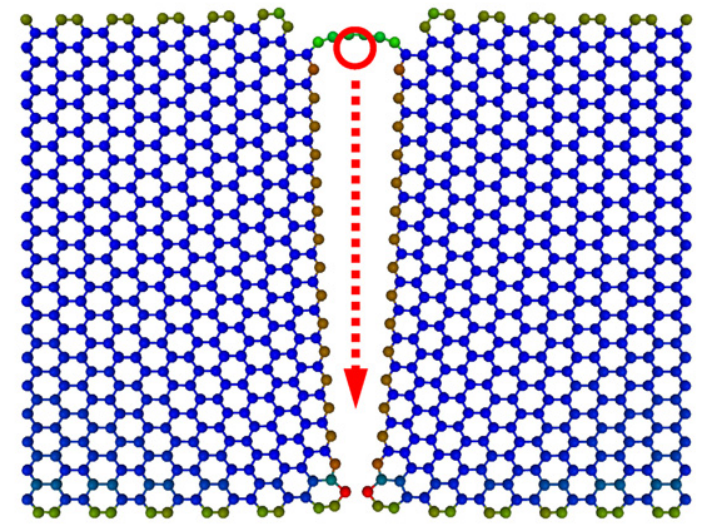

Figure 8. Fracture of GNRs under uniaxial tension. (a) Homogeneous nucleation for a zigzag GNR; $(b)$ edge-controlled heterogeneous nucleation for an armchair GNR. The circles indicate the nucleation sites, and the arrows indicate the directions of crack growth. Color indicates the potential energy of the carbon atoms.

stress for bulk graphene under uniaxial tension in the armchair direction (see figure 5(a)), in qualitative agreement with the MM calculations in this study. A more quantitative study on the fracture process of graphene is left for future work.

In addition to the fracture strain, the nominal fracture stress (i.e. uniaxial tensile strength) of the GNRs can be determined from the stress-strain curves in figure 4. As shown in figure 9, the fracture stress increases as the ribbon width increases for GNRs with unpassivated edges. The edge effect is relatively small for the zigzag-edged GNRs, with all the fracture stresses around $36 \mathrm{~N} \mathrm{~m}^{-1}$, very close to that of bulk graphene. For the armchair-edged GNRs, the fracture stress is considerably lower, e.g. $27.5 \mathrm{~N} \mathrm{~m}^{-1}$ for an unpassivated GNR with $W=2.5 \mathrm{~nm}$, compared with $30.6 \mathrm{~N} \mathrm{~m}^{-1}$ for bulk graphene under uniaxial tension in the armchair direction. Again, the lower fracture stress for the armchair-edged GNRs can be attributed to the edge-controlled heterogeneous nucleation mechanism shown in figure $8(b)$.

In this study we have focused on the fracture of defect-free GNRs. It is expected that interior defects of graphene lattice, such as vacancies, dislocations and grain boundaries, could have significant effects on the fracture of graphene. A similar effect has been studied for CNTs [48, 49]. Recently, Terdalkar et al [50] have presented atomistic simulations of the kinetic processes of bond breaking and bond rotation near a crack tip in graphene. Grantab et al [51] have demonstrated by atomistic calculations an anomalous effect of tilt grain 

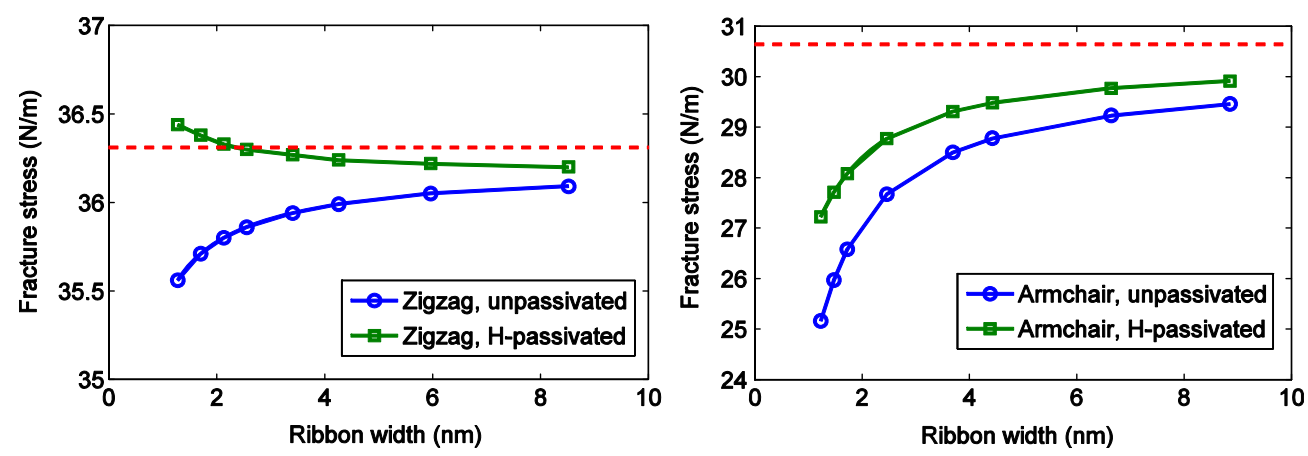

Figure 9. Nominal fracture stress versus ribbon width for GNRs under uniaxial tension, with $(a)$ zigzag and $(b)$ armchair edges. The horizontal dashed line in each figure indicates the fracture stress of bulk graphene under uniaxial tension in the same direction.

boundaries on the strength of graphene. Further studies on fracture of GNRs may consider interactions between the interior defects and the edge structures.

\section{Effects of hydrogen adsorption}

The edges of GNRs are often passivated with hydrogen $(\mathrm{H})$ atoms. Hydrogen adsorption changes the bonding environment and the energetics of the edges. Subject to uniaxial tension, the potential energy of a GNR now includes the contribution from hydrogen adsorption at the edges, namely

$$
\Phi(\varepsilon)=N U_{0}+U(\varepsilon) W L+2 \gamma(\varepsilon) L-2 \gamma_{\mathrm{H}}(\varepsilon) L,
$$

where $\gamma_{\mathrm{H}}(\varepsilon)$ is the adsorption energy per length for hydrogen passivated edges. The negative sign for the last term in equation (16) indicates typically reduced edge energy due to hydrogen adsorption $[17,23]$. By comparing the calculated potential energies for the GNRs with and without $\mathrm{H}$-passivation, the adsorption energy can be determined as a function of the nominal strain for both armchair and zigzag edges. At zero strain $(\varepsilon=0)$, our MM calculations predict the hydrogen adsorption energies to be $20.5 \mathrm{eV} \mathrm{nm}^{-1}$ and $22.6 \mathrm{eV} \mathrm{nm}^{-1}$ for the zigzag and armchair edges, respectively, which agree closely with the first-principles calculations [23]. Under uniaxial tension, the adsorption energy varies with the nominal strain, as shown in figure 10. The calculated H-adsorption energy is fitted with an eighth-order polynomial function, namely

$$
\gamma_{\mathrm{H}}(\varepsilon)=c_{0}+c_{1} \varepsilon+c_{2} \varepsilon^{2}+c_{3} \varepsilon^{3}+c_{4} \varepsilon^{4}+c_{5} \varepsilon^{5}+c_{6} \varepsilon^{6}+c_{7} \varepsilon^{7}+c_{8} \varepsilon^{8},
$$

where the coefficients are listed in table 3. The first three terms on the right-hand side of equation (17) directly affect the edge energy, edge force and edge modulus at infinitesimal strain, respectively, whereas the higher order terms account for the nonlinear effects with finite strain. The effect of elastic deformation (strain) on the adsorption energy demonstrates an intrinsic coupling between mechanics and chemistry on the atomistic scale.

The 2D nominal stress-strain relation for a GNR with H-passivated edges can then be obtained as

$$
\sigma(\varepsilon)=\frac{\mathrm{d} U}{\mathrm{~d} \varepsilon}+\frac{2}{W}\left(\frac{\mathrm{d} \gamma}{\mathrm{d} \varepsilon}-\frac{\mathrm{d} \gamma_{\mathrm{H}}}{\mathrm{d} \varepsilon}\right)
$$




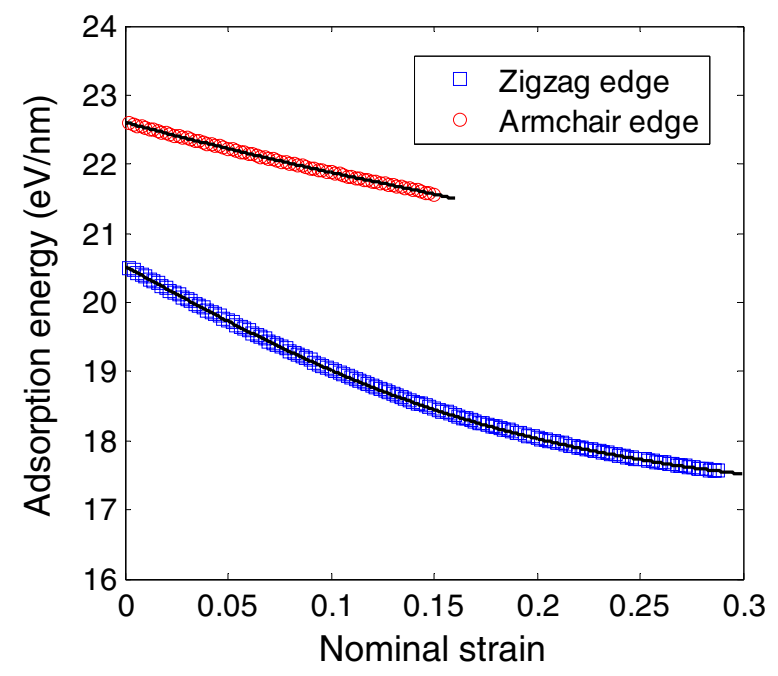

Figure 10. Hydrogen adsorption energy of GNRs under uniaxial tension. The open symbols are obtained directly from atomistic simulations, and the solid lines are the polynomial fitting in equation (17).

Table 3. Coefficients of the polynomial fitting in equation (17) for the hydrogen adsorption energy of GNRs with zigzag and armchair edges (unit: $\mathrm{eV} \mathrm{nm}^{-1}$ ).

\begin{tabular}{lll}
\hline & Zigzag & Armchair \\
\hline$c_{0}$ & 20.53 & 22.61 \\
$c_{1}$ & -16.14 & -8.25 \\
$c_{2}$ & 0.3798 & 21.66 \\
$c_{3}$ & 144.49 & -297.69 \\
$c_{4}$ & -577.04 & 1755.68 \\
$c_{5}$ & 5109.55 & 19851.33 \\
$c_{6}$ & -31512.41 & -342205.25 \\
$c_{7}$ & 84961.78 & 1835393.08 \\
$c_{8}$ & -83158.52 & -3589655.15 \\
\hline
\end{tabular}

and the tangent modulus is

$$
E(\varepsilon)=\frac{\mathrm{d}^{2} U}{\mathrm{~d} \varepsilon^{2}}+\frac{2}{W}\left(\frac{\mathrm{d}^{2} \gamma}{\mathrm{d} \varepsilon^{2}}-\frac{\mathrm{d}^{2} \gamma_{\mathrm{H}}}{\mathrm{d} \varepsilon^{2}}\right) .
$$

Figure 11 compares the stress-strain curves for H-passivated GNRs, unpassivated GNRs and bulk graphene. At infinitesimal strain, the initial Young's modulus follows equation (12), but with a modified edge modulus due to $\mathrm{H}$-adsorption, namely

$$
E_{0}^{\mathrm{e}}=\left(\frac{\mathrm{d}^{2} \gamma}{\mathrm{d} \varepsilon^{2}}\right)_{\varepsilon=0}-\left(\frac{\mathrm{d}^{2} \gamma_{\mathrm{H}}}{\mathrm{d} \varepsilon^{2}}\right)_{\varepsilon=0}=2 b_{2}-2 c_{2} .
$$

As shown in figure 6, H-adsorption has a negligible effect on the initial Young's modulus for GNRs with zigzag edges. In contrast, the effect is significant for GNRs with armchair edges. The edge modulus as defined in equation (20) becomes negative for the H-passivated armchair edge. Consequently, by equation (12), the initial Young's modulus of the GNR decreases as the ribbon width decreases, opposite to the unpassivated GNRs. 

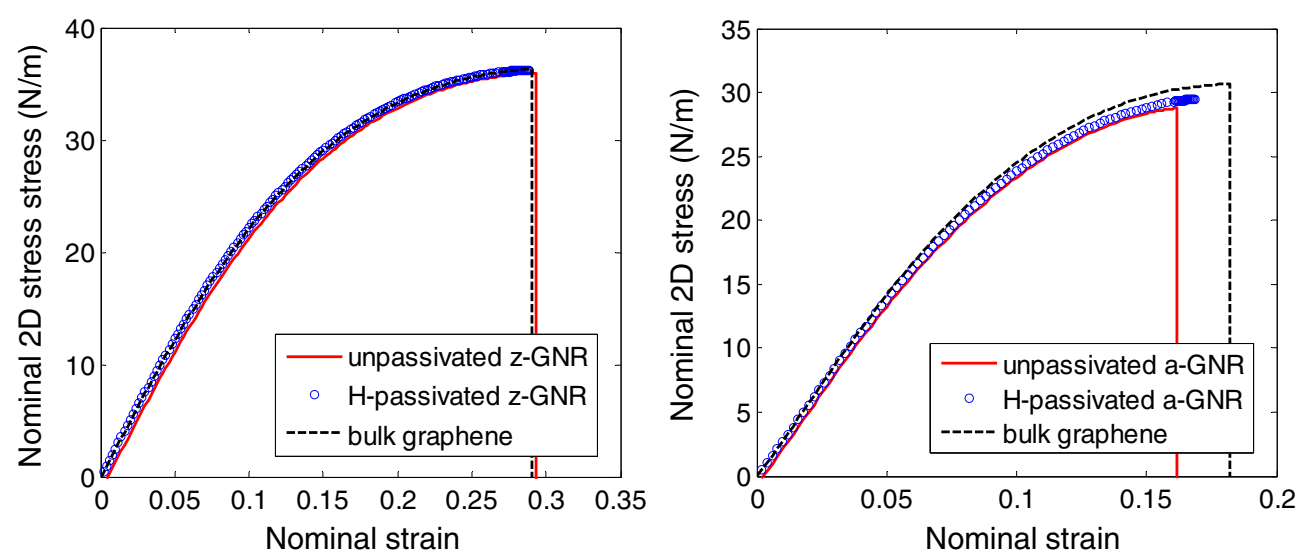

Figure 11. Comparison of nominal stress-strain curves under uniaxial tension for bulk graphene, GNRs with unpassivated edges and GNRs with hydrogen-passivated edges: $(a)$ zigzag-edged GNR $(W=4.3 \mathrm{~nm})$ and $(b)$ armchair-edged GNR $(W=4.4 \mathrm{~nm})$.

The effect of hydrogen adsorption on fracture strain is shown in figure 7. Hydrogen passivation of the edges leads to slightly lower fracture strains for zigzag GNRs, but slightly higher fracture strains for armchair GNRs. The effect is relatively small in both cases. Figure 9 shows that $\mathrm{H}$-adsorption slightly increases the fracture stress for both zigzag- and armchairedged GNRs. The same facture mechanisms shown in figure 8 are observed in MD simulations for GNRs with H-passivated edges.

\section{Summary}

This paper presents a theoretical study on the effects of edge structures on the mechanical properties of graphene nanoribbons (GNRs) under uniaxial tension. Both the bulk strain energy density and edge energy density (without and with hydrogen passivation) are calculated from atomistic simulations as functions of the nominal strain. Due to the edge effect, the initial Young's modulus of GNRs under infinitesimal strain depends on both the chirality and ribbon width. Furthermore, it is found that the strain to fracture is considerably lower for armchair-edged GNRs than for zigzag-edged GNRs. Two distinct fracture nucleation mechanisms are identified, homogeneous nucleation for GNRs with zigzag edges and edgecontrolled heterogeneous nucleation for those with armchair edges. Hydrogen adsorption along the edges is found to have relatively small effects on the mechanical behavior of zigzagedged GNRs, but its effect is more significant for armchair-edged GNRs. Finally, we note that several reconstructions have been predicted for the edge structures of graphene [23,46, 47] and reconstructed edges may have different effects on the mechanical properties of GNRs [11] compared with the pristine edges considered in this study.

\section{Acknowledgments}

The authors gratefully acknowledge funding of this work by the National Science Foundation through Grant No 0926851. They thank Dr Jeffrey W Kysar of Columbia University for helpful discussions and for providing the first-principles results for comparison in figure 5. 


\section{References}

[1] Berger C, Song Z M, Li X B, Wu X S, Brown N, Naud C, Mayou D, Li T B, Hass J and Marchenkov A N 2006 Electronic confinement and coherence in patterned epitaxial graphene Science 312 1191-6

[2] Ozyilmaz B, Jarillo-Herrero P, Efetov D, Abanin D A, Levitov L S and Kim P 2007 Electronic transport and quantum Hall effect in bipolar graphene p-n-p junctions Phys. Rev. Lett. 99166804

[3] Lin Y M, Perebeinos V, Chen Z H and Avouris P 2008 Electrical observation of subband formation in graphene nanoribbons Phys. Rev. B $78161409 \mathrm{R}$

[4] Li X L, Wang X R, Zhang L, Lee S W and Dai H J 2008 Chemically derived, ultrasmooth graphene nanoribbon semiconductors Science 319 1229-32

[5] Nakada K, Fujita M, Dresselhaus G and Dresselhaus M S 1996 Edge state in graphene ribbons: nanometer size effect and edge shape dependence Phys. Rev. B 54 17954-61

[6] Son Y-W, Cohen M L and Louie S G 2006 Energy gaps in graphene nanoribbons Phys. Rev. Lett. 97216803

[7] Barone V, Hod O and Scuseria G E 2006 Electronic structure and stability of semiconducting graphene nanoribbons Nano Lett. 6 2748-54

[8] Dutta S, Lakshmi S and Pati S K 2008 Electron-electron interactions on the edge states of graphene: a many-body configuration interaction study Phys. Rev. B 77073412

[9] Shenoy V B, Reddy C D, Ramasubramaniam A and Zhang Y W 2008 Edge-stress-induced warping of graphene sheets and nanoribbons Phys. Rev. Lett. 101245501

[10] Bets K V and Yakobson B I 2009 Spontaneous twist and intrinsic instabilities of pristine graphene nanoribbons Nano Res. 2 161-6

[11] Reddy C D, Ramasubramaniam A, Shenoy V B and Zhang Y-W 2009 Edge elastic properties of defect-free single-layer graphene sheets Appl. Phys. Lett. 94101904

[12] Zhao H, Min K and Aluru N R 2009 Size and chirality dependent elastic properties of graphene nanoribbons under uniaxial tension Nano Lett. $93012-5$

[13] Bu H, Chen Y F, Zou M, Yi H, Bi K and Ni Z 2009 Atomistic simulations of mechanical properties of graphene nanoribbons Phys. Lett. A 373 3359-62

[14] Xu Z P 2009 Graphene nano-ribbons under tension J. Comput. Theor. Nanosci. 6 625-8

[15] Faccio R, Denis P A, Pardo H, Goyenola C and Mombru A W 2009 Mechanical properties of graphene nanoribbons J. Phys.: Condens. Matter 21285304

[16] Topsakal M and Ciraci S 2010 Elastic and plastic deformation of graphene, silicene, and boron nitride honeycomb nanoribbons under uniaxial tension: a first-principles density-functional theory study Phys. Rev. B 81024107

[17] Gan C K and Srolovitz D J 2010 First-principles study of graphene edge properties and flake shapes Phys. Rev. B 81125445

[18] Lu Q and Huang R 2010 Excess energy and deformation along free edges of graphene nanoribbons Phys. Rev. B 81155410

[19] Liu F, Ming P M and Li J 2007 Ab initio calculation of ideal strength and phonon instability of graphene under tension Phys. Rev. B 76064120

[20] Wei X, Fragneaud B, Marianetti C A and Kysar J W 2009 Nonlinear elastic behavior of graphene: ab initio calculations to continuum description Phys. Rev. B 80205407

[21] Lu Q and Huang R 2009 Nonlinear mechanics of single-atomic-layer graphene sheets Int. J. Appl. Mech. $1443-67$

[22] Lee C, Wei X, Kysar J W and Hone J 2008 Measurement of the elastic properties and intrinsic strength of monolayer graphene Science $321385-8$

[23] Koskinen P, Malola S and Hakkinen H 2008 Self-passivating edge reconstructions of graphene Phys. Rev. Lett. 101115502

[24] Jia X et al 2009 Controlled formation of sharp zigzag and armchair edges in graphitic nanoribbons Science 323 1701-5

[25] Yu M F, Lourie O, Dyer M J, Moloni K, Kelly T F and Ruoff R S 2000 Strength and breaking mechanism of multiwalled carbon nanotubes under tensile load Science $287637-40$

[26] Brenner D W, Shenderova O A, Harrison J A, Stuart S J, Ni B and Sinnott S B 2002 A second-generation reactive empirical bond order (REBO) potential for hydrocarbons J. Phys.: Condens. Matter 14 783-802

[27] Lu Q, Arroyo M and Huang R 2009 Elastic bending modulus of monolayer graphene J. Phys. D: Appl. Phys. 42102002

[28] Shenderova O A, Brenner D W, Omeltchenko A, Su X and Yang L H 2000 Atomistic modeling of the fracture of polycrystalline diamond Phys. Rev. B $613877-88$

[29] Belytschko T, Xiao S P, Schatz G C and Ruoff R S 2002 Atomistic simulations of nanotube fracture Phys. Rev. B $\mathbf{6 5} 235430$ 
[30] Zhang P, Jiang H, Huang Y, Geubelle P H and Hwang K C 2004 An atomistic-based continuum theory for carbon nanotubes: analysis of fracture nucleation J. Mech. Phys. Solids 52 977-98

[31] Liu D C and Nocedal J 1989 On the limited memory BFGS method for large scale optimization Math. Program. B 45 503-28

[32] Andersen H C 1980 Molecular dynamics at constant pressure and/or temperature J. Chem. Phys. 72 2384-93

[33] Aitken Z H and Huang R 2010 Effects of mismatch strain and substrate surface corrugation on morphology of supported monolayer graphene J. Appl. Phys. 107123531

[34] Arroyo M and Belytschko T 2004 Finite crystal elasticity of carbon nanotubes based on the exponential CauchyBorn rule Phys. Rev. B 69115415

[35] Huang Y, Wu J and Hwang K C 2006 Thickness of graphene and single-wall carbon nanotubes Phys. Rev. B 74245413

[36] Zhou J and Huang R 2008 Internal lattice relaxation of single-layer graphene under in-plane deformation J. Mech. Phys. Solids 56 1609-23

[37] Stuart S J, Tutein A B and Harrison J A 2000 A reactive potential for hydrocarbons with intermolecular interactions J. Chem. Phys. 112 6472-86

[38] Tewary V K and Yang B 2009 Parametric interatomic potential for graphene Phys. Rev. B 79075442

[39] Lindsay L and Briodo D A 2010 Optimized Tersoff and Brenner empirical potential parameters for lattice dynamics and phonon thermal transport in carbon nanotubes and graphene Phys. Rev. B 81205441

[40] Dingreville R, Qu J and Cherkaoui M 2005 Surface free energy and its effect on the elastic behavior of nano-sized particles, wires and films J. Mech. Phys. Solids 53 1827-54

[41] Cao G and Chen X 2007 Energy analysis of size-dependent elastic properties of ZnO nanofilms using atomistic simulations Phys. Rev. B 76165407

[42] Huang R, Stafford C M and Vogt B D 2007 Effect of surface properties on wrinkling of ultrathin films J. Aerospace Eng. $2038-44$

[43] Marianetti C A and Yevick H G 2010 Failure mechanisms of graphene under tension arXiv:1004.1849v1

[44] Jin C, Lan H, Peng L, Suenaga K and Iijima S 2009 Deriving carbon atomic chains from graphene Phys. Rev. Lett. 102205501

[45] Lawn B 1993 Fracture of Brittle Solids 2nd edn (Cambridge: Cambridge University Press)

[46] Jun S 2008 Density-functional study of edge stress in graphene Phys. Rev. B 78073405

[47] Huang B, Liu M, Su N, Wu J, Duan W, Gu B and Liu F 2009 Quantum manifestations of graphene edge stress and edge instability: a first-principles study Phys. Rev. Lett. 102166404

[48] Mielke S L, Troya D, Zhang S, Li J-L, Xiao S, Car R, Ruoff R S, Schatz G C and Belytschko T 2004 The role of vacancy defects and holes in the fracture of carbon nanotubes Chem. Phys. Lett. 390 413-20

[49] Zhang S, Mielke S L, Khare R, Troya D, Ruoff R S, Schatz G C and Belytschko T 2005 Mechanics of defects in carbon nanotubes: atomistic and multiscale simulations Phys. Rev. B 71115403

[50] Terdalkar S S, Huang S, Yuan H, Rencis J J, Zhu T and Zhang S 2010 Nanoscale fracture in graphene Chem. Phys. Lett. 494 218-22

[51] Grantab R, Shenoy V B and Ruoff R S 2010 Anomalous strength characteristics of tilt grain boundaries in graphene Science 330 946-8 